\title{
Electronic pillbox-enabled self-administered therapy versus standard directly observed therapy for tuberculosis medication adherence and treatment outcomes in Ethiopia (SELFTB): protocol for a multicenter randomized controlled trial
}

Tsegahun Manyazewal ${ }^{1 *}$ (D), Yimtubezinash Woldeamanuel ${ }^{1}$, David P. Holland², Abebaw Fekadu', Henry M. Blumberg ${ }^{2}$ and Vincent C. Marconi ${ }^{2}$

\footnotetext{
Abstract

Background: To address the multifaceted challenges associated with tuberculosis (TB) in-person directly observed therapy (DOT), the World Health Organization recently recommended that countries maximize the use of digital adherence technologies. Sub-Saharan Africa needs to investigate the effectiveness of such technologies in local contexts and proactively contribute to global decisions around patient-centered TB care. This study aims to evaluate the effectiveness of pillbox-enabled self-administered therapy (SAT) compared to standard DOT on adherence to TB medication and treatment outcomes in Ethiopia. It also aims to assess the usability, acceptability, and cost-effectiveness of the intervention from the patient and provider perspectives.

(Continued on next page)
}

\footnotetext{
${ }^{*}$ Correspondence: tsegahunm@gmail.com

'Addis Ababa University, College of Health Sciences, Center for Innovative Drug Development and Therapeutic Trials for Africa, P.O. Box 9086, Addis Ababa, Ethiopia

Full list of author information is available at the end of the article
}

(c) The Author(s). 2020 Open Access This article is licensed under a Creative Commons Attribution 4.0 International License, which permits use, sharing, adaptation, distribution and reproduction in any medium or format, as long as you give appropriate credit to the original author(s) and the source, provide a link to the Creative Commons licence, and indicate if changes were made. The images or other third party material in this article are included in the article's Creative Commons licence, unless indicated otherwise in a credit line to the material. If material is not included in the article's Creative Commons licence and your intended use is not permitted by statutory regulation or exceeds the permitted use, you will need to obtain permission directly from the copyright holder. To view a copy of this licence, visit http://creativecommons.org/licenses/by/4.0/. The Creative Commons Public Domain Dedication waiver (http://creativecommons.org/publicdomain/zero/1.0/) applies to the data made available in this article, unless otherwise stated in a credit line to the data. 
(Continued from previous page)

Methods: This is a multicenter, randomized, controlled, open-label, superiority, effectiveness-implementation hybrid, mixed-methods, two-arm trial. The study is designed to enroll 144 outpatients with new or previously treated, bacteriologically confirmed, drug-sensitive pulmonary TB who are eligible to start the standard 6-month first-line anti-TB regimen. Participants in the intervention arm $(n=72)$ will receive 15 days of HRZE-isoniazid, rifampicin, pyrazinamide, and ethambutol-fixed-dose combination therapy in the evriMED500 medication event reminder monitor device for self-administration. When returned, providers will count any remaining tablets in the device, download the pill-taking data, and refill based on preset criteria. Participants can consult the provider in cases of illness or adverse events outside of scheduled visits. Providers will handle participants in the control arm ( $\mathrm{n}=72$ ) according to the standard in-person DOT. Both arms will be followed up throughout the 2-month intensive phase. The primary outcomes will be medication adherence and sputum conversion. Adherence to medication will be calculated as the proportion of patients who missed doses in the intervention (pill count) versus DOT (direct observation) arms, confirmed further by IsoScreen urine isoniazid test and a self-report of adherence on eight-item Morisky Medication Adherence Scale. Sputum conversion is defined as the proportion of patients with smear conversion following the intensive phase in intervention versus DOT arms, confirmed further by pre-post intensive phase BACTEC MGIT TB liquid culture. Pre-post treatment MGIT drug susceptibility testing will determine whether resistance to anti-TB drugs could have impacted culture conversion. Secondary outcomes will include other clinical outcomes (treatment not completed, death, or loss to follow-up), cost-effectiveness-individual and societal costs with quality-adjusted life years - and acceptability and usability of the intervention by patients and providers.

Discussion: This study will be the first in Ethiopia, and of the first three in sub-Saharan Africa, to determine whether electronic pillbox-enabled SAT improves adherence to TB medication and treatment outcomes, all without affecting the inherent dignity and economic wellbeing of patients with TB.

Trial registration: ClinicalTrials.gov, NCT04216420. Registered on 2 January 2020.

Keywords: Tuberculosis, Pillbox, Self-administered therapy, Directly observed therapy (DOT), Adherence, Treatment outcome, Trials, Sub-Saharan Africa, Ethiopia

\section{Administrative information}

\begin{tabular}{ll}
\hline Title & Electronic pillbox-enabled self- \\
& administered therapy versus standard dir- \\
& ectly observed therapy for tuberculosis \\
& medication adherence and treatment \\
& outcomes in Ethiopia (SELFTB): protocol \\
& for a multicenter randomized controlled \\
& trial
\end{tabular}

Trial registration

Protocol version:

Funding:

Author details:

\section{Administrative information (Continued)}

${ }^{2}$ Emory University School of Medicine and Rollins School of Public Health, Atlanta, Georgia, 30322, United States of America

Study conception and Principal Investigator: TM. Initial study design and protocol: TM. Major contribution to study design and protocol: VCM, YW, DPH. Resource acquisition: HMB, AF. Draft the manuscript: TM. Reviewed and revised the manuscript: TM, VCM, YW, DPH, HMB, AF. Read and approved the final manuscript: TM, VCM, YW, DPH, HMB, AF.

Name and contact information for the trial sponsor:

Addis Ababa University - Email: health. sciences@aau.edu.et, Phone:

+251118959055, website: www.aau.edu. et, P.O. Box: 9086, Addis Ababa, Ethiopia. The information and views set out in this study are those of the authors and do not necessarily reflect the official opinion of the Addis Ababa University

\section{Introduction}

The World Health Organization (WHO) revealed its commitment to patients with tuberculosis (TB) in its End TB strategy that "everyone with TB should have access to the innovative tools and services they need for rapid diagnosis, treatment, and care; this is a matter of 
social justice, fundamental to our goal of universal health coverage." This commitment is a collective responsibility towards human rights, making sure that no family is burdened with avoidable death or catastrophic expenses due to TB by 2030. However, taking pride in the slogan "End TB by 2030" is insufficient for true progress. In order to translate aspirational goals into reality, practical solutions should be sought. The strategy might appear to be a zero-sum if interventions on the disease neglect key socioeconomic burdens that individual patients are incapable of avoiding. The association between TB and poverty is a reality [1-5], but the disease is a global health security threat that urgently needs collective resources for mutual welfare $[6,7]$. In the last three decades, various strategies and intervention packages have been formulated and implemented to halt the disease. However, improvements are not as expected [8, 9]; instead, a drugresistant form of the disease is spreading [10-12], with globalization and migration fueling multiple strains worldwide [13-15]. TB remains the top cause of death worldwide from a single infectious disease and the major cause of death due to antimicrobial resistance. According to the 2019 WHO global TB report, an estimated 10.0 million people fell ill and 1.5 million people died from TB in 2018. Drug-resistant TB (DR-TB) continues to be a public health threat, where there were about half a million new cases of rifampicin-resistant $\mathrm{TB}$, of which $78 \%$ had multidrug-resistant TB [16]. Africa accounts for one-quarter of new TB cases and TB-related deaths worldwide, with 2.5 million people falling ill and 417,000 people dying from TB annually. Of the total patients with TB co-infected with HIV globally, $72 \%$ of them live in Africa [17]. The true burden of DR-TB in the continent is poorly described, with only $51 \%$ of countries having formal data in the WHO global TB database, where DR-TB is largely missed and this requires a major effort to achieve the 2035 targets [16].

The main challenge is how to meet the End TB's vision of "A world free of TB: zero deaths, disease and suffering due to TB" without bargaining the inherent dignity and economic wellbeing of patients with TB. It remains unclear how resource-limited countries would be able to meet one of the four key indicators of the strategy "Zero TB-affected families facing catastrophic costs due to TB by 2035 " in situations where management of TB treatment still relies on directly observed therapy (DOT). Patients with TB from the poorer segments of society may not be benefiting from care delivery innovations: an apparent contradiction between "Global Commitment to End TB" and "reality on the ground." Management of TB still largely depends on DOT. DOT has been viewed as an efficient strategy for adherence to treatment $[18,19]$, while evidence has demonstrated that it poses an economic and social burden to patients with TB and healthcare programs from low-income countries [20-25]. Treatment of TB requires at least 6 months, where patients in the intensive phase of DOT need to collect their medication at healthcare facilities daily and swallow tablets under the direct observation of a healthcare worker throughout the intensive phase [20,21, 26].

To address the multifaceted problems associated with TB in-person DOT, the WHO recently recommended countries to maximize the use of digital adherence technologies (DATs). Such technologies, in general, have been able to improve TB treatment outcomes [27-30] and substantially reduce costs [31]. Yet, available data are limited for better conclusions of their effectiveness in various countries and settings [32-35]. In 2017, the WHO endorsed three DATs: short message service/mobile phone texting (SMS); Medication Event Reminder Monitor System (MERM); and video-supported directly observed therapy (VDOT) [36]. SMS involves sending a standardized and understandable text message to patients with TB regularly to remind and motivate them to take their prescribed medications. MERM is an electronic pillbox that records adherence to treatment, stores medication, emits audible and visual alerts to remind patients to take their medications, and enables healthcare providers to monitor adherence. MERM sleeves (99DOTS prototype) have an additional component that each medication blister is wrapped in 99DOTS envelopes to send to providers a hidden signal unrecognized by the patient. VDOT involves video communication between patients and healthcare providers, where providers watch patients take their medication, live or self-recorded, and provide advice and support. VDOT is mediated primarily through Internet-enabled smartphones, and Internet access is a critical component [36]. In general, there have been limited studies conducted on DATs. For existing studies, effectiveness within various low- and middle-income countries (LMIC) has been the focus of research and has resulted in some controversy. Table 1 summarizes a review of recent studies conducted on the three DATs.

Considering the low-impact of SMS for adherence to $\mathrm{TB}$ treatment and the high investment and technology needed for VDOT, the advantage of MERM electronic pillboxes could surpass the other technologies, though more studies from LMIC are needed to inform its effectiveness. There are many electronic pillbox devices available in the global market; several were not endorsed by international health stakeholders (e.g. the WHO) or are not accessible in LMICs. Similar pillboxes have been in use for several decades for various medications. With the use of such an ordinary pillbox, patients can selfmanage their medications, identify whether they have 
Table 1 Summary of existing literature on TB digital adherence technologies

\begin{tabular}{|c|c|c|c|c|}
\hline Reference & Country & Design & Outcome measure & Finding \\
\hline \multicolumn{5}{|l|}{ SMS } \\
\hline Bediang et al. 2018 [37] & Cameroon & RCT: SMS vs DOT & Treatment success, cure & No significant difference \\
\hline Fang et al. 2017 [38] & China & RCT: SMS vs DOT & Treatment completion & High \\
\hline Mohammed et al. 2016 [39] & Pakistan & RCT: SMS vs DOT & Treatment success, cure & No significant difference \\
\hline Liu et al. 2015 [40] & China & RCT: 4 arms & Pill count, adherence & No significant improvement \\
\hline Iribarren et al. 2013 [41] & Argentina & Cross-sectional & Feasibility and acceptability & acceptable and feasible \\
\hline \multicolumn{5}{|l|}{ MERM } \\
\hline Onwubiko et al. 2019 [42] & USA & RCT: MERM vs DOT & Treatment completion & Low \\
\hline Park et al. 2019 [43] & Morocco & RCT: MERM vs DOT & Treatment success, cure & High \\
\hline Liu et al. 2017 [44] & China & Multi-method & User performance, satisfaction & High \\
\hline Broomhead et al. 2012 [45] & USA & Cross-sectional & Treatment outcome, cost & High, lower cost per patient \\
\hline Thakkar et al. 2019 [46] & India & Cohort, 99DOTS used & Treatment adherence & High \\
\hline \multicolumn{5}{|l|}{ VDOT } \\
\hline Lam et al. 2018 [47] & USA & RCT. VDOT vs DOT & Treatment completion & High \\
\hline Garfein et al. 2018 [48] & USA & RCT: VDOT vs DOT & Adherence, cost & High, lower cost \\
\hline Nguyen et al. 2017 [49] & Vietnam & Prospective cohort & Treatment adherence & High \\
\hline Chuck et al. 2016 [50] & USA & RCT: VDOT vs DOT & Treatment completion & High \\
\hline Garfein et al. 2015 [51] & USA, Mexico & Single-arm trial & Treatment adherence & High in both settings \\
\hline
\end{tabular}

DOT directly observed therapy, $R C T$ randomized control trial, VDOT video directly observed therapy

taken the dose, and minimize the rate of medication errors [52]. Previous studies found that individuals who used a pillbox had better adherence to treatment [53-55].

The evriMed500, manufactured by Wisepill Technologies, South Africa, is among the MERMs available on the market. The device is TB-appropriate technology allowing customization of the container for patients with drugsusceptible TB, MDR-TB, and TB-HIV [56]. It is currently being tested in several clinical trials and is being used clinically in India and China for patients with TB [57]. It costs less than US\$10 per patient based on conservative reuse assumptions [58]. The evriMED500 dispenser consists of an electronic module and a medication container with three indicator lights/LEDs (green, yellow, and red). The green LED will flash once when the container is opened and again once when the container is closed, will quickly flash three times when the container is opened and closed quickly, will flash in sequence during the (daily) Medication Alarm, and will be on solid while connected via USB to a computer. The yellow LED indicated a need to refill medications and will flash with the green LED at the time of the Medication Alarm. If the Medication Alarm is not enabled, only the yellow LED will flash. The yellow LED will be on solid when the container is opened. The red LED flash indicates low battery power and will flash with the green LED at the time of the Medication Alarm. The red LED will be on solid when the container is opened. The device requires two AA batteries, which should last for more than 12 months. It can store more than 12,000 records/events.

In-person DOT is highly challenging for most patients with TB in sub-Saharan Africa. In these settings, DOT did not provide a significant solution for suboptimal treatment adherence. Instead, home-based and community-based therapies were shown to be possible alternative strategies to health-facility DOT according to studies conducted in Tanzania [59-61], Kenya [62, 63], Zambia [64], South Africa [65, 66], and Eritrea [67].

Ethiopia is among several countries highly encumbered by the TB epidemic and one of the least resourced in the world. According to the 2019 global TB report of the WHO, there were $113,613 \mathrm{~TB}$ cases reported in the country; and of the annual \$94 million needed for TB care and control, the country's domestic contribution was only $11 \%$ of this [17]. Despite TB care and treatment services being delivered free of charge, patients with TB face out-of-pocket payments [68, 69] and income losses [70] due to transportation, accommodation, and food to get treatment at a healthcare facility. This can be a major obstacle to adherence and has forced patients to stop working, sell their property, borrow money, and reduce their overall income [71]. These, in turn, have increased rates of loss to follow-up, disease relapse, and drug resistance [72]. Patients who travel daily to healthcare facilities for medication have also increased the transmission potential of the disease, especially in the capital city Addis 
Ababa, a highly congested city with overcrowded housing and public transportation [73]. Several studies conducted in Addis Ababa reported that patients with TB consider their daily DOT visits as worthless [74-78], and providers see DOT as a very challenging strategy for patients with TB [76]. As a result, daily DOT survives in principle, while implementation is irregular as both patients with $\mathrm{TB}$ and providers have uncertainties concerning the program. Providers report that patients with TB prefer taking the tablets at home once they have the necessary advice and counseling [74, 76]. Patients complain that they travel for daily DOT on foot under harsh road conditions for up to $2 \mathrm{~h}$ and $2.5 \mathrm{~km}$, taking several rests on their way because of their sickness [74, 77, 79]. They spend substantial money on transportation $[76,80]$ and some lose their job due to work absences related to daily DOT [74, 78, 79]. Providers also report that patients appear exhausted and dissatisfied during DOT visits [74, 78]. Some patients claim that once they are initially informed of the disease, no-one talks with them on the subsequent DOT days; they just swallow the drug and return home [76]. Additionally, patients face stigma on their route to daily DOT and change their name in the TB clinic to disguise their identity [80]. The challenge is similar in other parts of Ethiopia [70, 81-83]. Such evidence discloses the difficulty of Ethiopia to meeting the overall End TB strategy targets by 2035 [84] unless significant investment is made to shape the current DOT strategy to a more advanced, technology-led, patientcentered strategy that could be responsive to individual patient preferences, needs, and values.

\section{Objectives}

The main aim of this trial is to evaluate the effectiveness of pillbox-enabled SAT over standard DOT on adherence to TB medication and treatment outcomes in Ethiopia-a high TB burden country in sub-Saharan African. The secondary objectives are to assess the costeffectiveness, thus individual and societal costs of treatment and quality-adjusted life years (QALY), when management of adherence followed pillbox-enabled SAT versus the standard DOT, and to assess the usability and acceptability by patients and their healthcare providers of the pillbox-enabled SAT.

\section{Methods}

\section{Trial design}

The study will be a prospective, multicenter, open-label, randomized, controlled, superiority, effectivenessimplementation hybrid, mixed-methods, two-arm trial. The study will not dictate diagnosis or treatment for TB; thus, it will not introduce or use new medications. The content of this protocol complies with the Standard Protocol Items: Recommendations for Interventional
Trials (SPIRIT) guidelines [85] (see Supplementary file 1). Figure 1 is the SPIRIT figure summarizing the overall schedule and design of the trial.

\section{Study setting}

Ethiopia is structurally divided into 11 autonomous administrative divisions. Addis Ababa, the capital, is among these administrative divisions and is the largest city of Ethiopia with the status of both a city and state. Addis Ababa is considered by some to be the capital of Africa, as it is a seat for the African Union headquarters and other international and regional organizations including the Africa Centers for Disease Control and Prevention. Administratively, Addis Ababa is divided into 10 sub-cities with distinct locations. As the city is crowded with population and housing, there is a high risk of TB transmission. The national health and healthrelated indicator [86] reports that in 2016, 2290 bacteriologically confirmed TB cases were identified in the city, of which 1811 (79.1\%) completed their treatment and 1526 (66.6\%) were cured. For these reasons, Addis Ababa was chosen as the study setting.

In Addis Ababa, a total of 94 public health centers provide TB care and treatment services under the DOT program. This study stratifies the 94 health centers on the bases of the 10 sub-cities where they are located. From each stratified group, one health center with the largest TB client load will be selected, with a total of 10 health centers to be included. Health management information system (HMIS) quarterly data (1 April-30 June 2019) taken from the Addis Ababa Regional Health Bureau will be used for the purpose. This will give a representative sample of study sites and participants. A TB clinic in each center will serve as the study site and primary location for patient contact for that center. Table 2 lists the 10 public health centers.

\section{Eligibility criteria}

The source population will be all new patients with TB symptoms who come to a study site and undergo bacteriological screening during the study period or TB patients bacteriologically confirmed elsewhere and referred to a study site for TB treatment.

Inclusion criteria will include:

- Patients with new, or previously treated, bacteriologically confirmed, drug-sensitive pulmonary $\mathrm{TB}$

- Eligible to start the standard 6-month first-line antiTB medication

- Outpatient status at the time of screening and enrollment

- Men or women aged 18-75 years, inclusive

- Able and willing to provide informed consent 


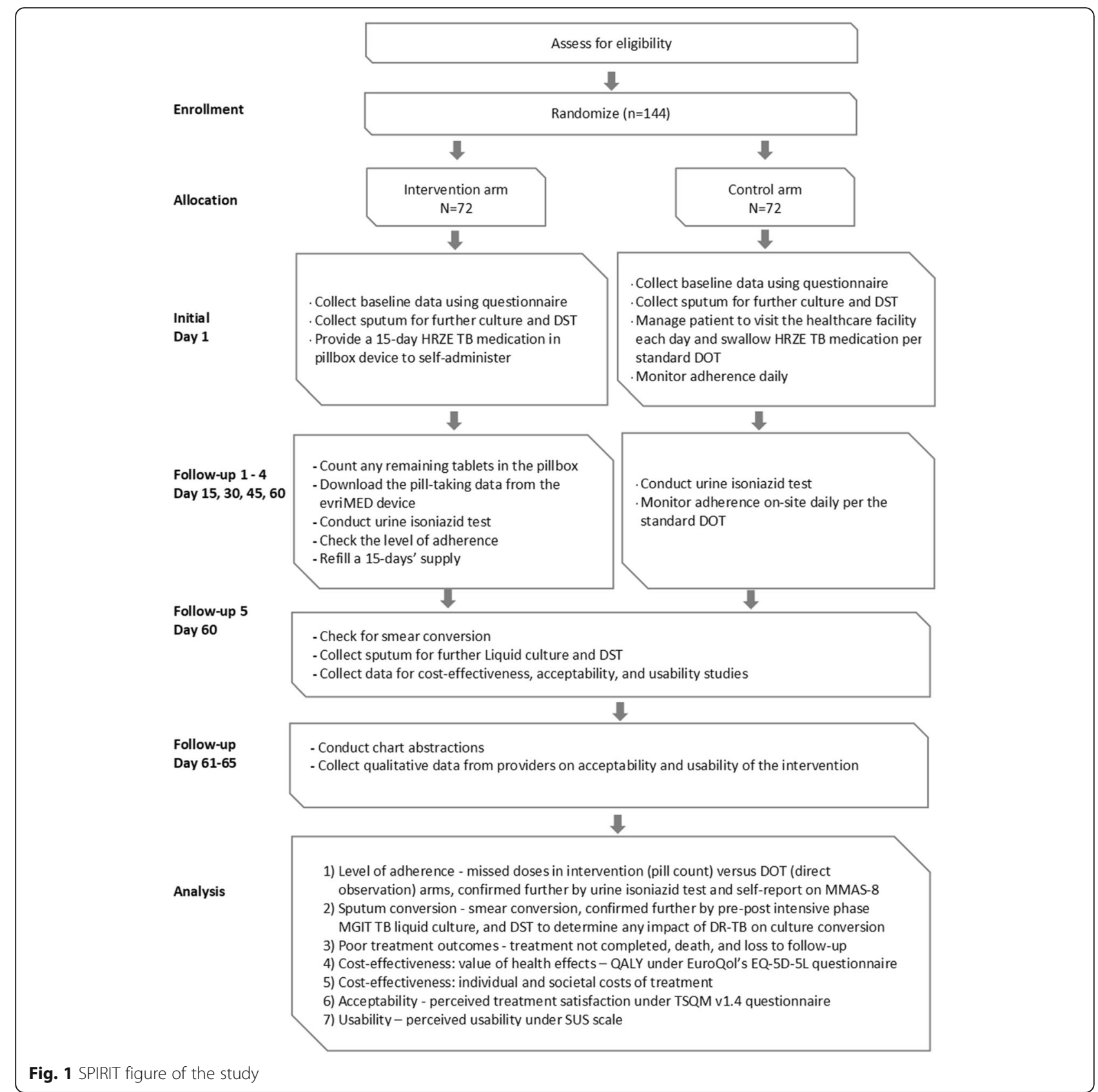

The exclusion criteria will include:

- Patients with known DR-TB

- Any condition that causes cognitive impairment such as severe acute illness or injury, developmental retardation, or severe psychiatric illness and thus precludes informed consent or safely participating in the study procedures

- Inpatient status at the time of screening and enrollment

- Expected to move away from the study site or become incarcerated before the final study follow-up at month 2
- Concurrent extra-pulmonary TB

- Contraindicated medications

- Active liver disease that requires a TB regimen other than HREZ (isoniazid, rifampicin, pyrazinamide, and ethambutol)

The calculated total sample size is 144 participants with 72 in each arm. Considering a two-sided type-I error of $5 \%$, probability of a type-II error of $20 \%$, a power of $80 \%, 5 \%$ missing outcome data, and $\pm 10 \%$ superiority from the current estimated adherence rate of 
Table 2 Study sites

\begin{tabular}{|c|c|c|c|}
\hline No. & Name of Health Center & Sub-city & $\begin{array}{l}\text { No of. PTB cases } \\
\text { bacteriologically } \\
\text { confirmed or referred } \\
\text { from other sites for } \\
\text { initiation of TB treatment, } \\
1 \text { April-30 June } 2019\end{array}$ \\
\hline & Addis Raey Health Center & Addisketema & 41 \\
\hline & Akaki Health Center & Akaki kality & 47 \\
\hline & Kebena Health Center & Arada & 14 \\
\hline & Goro Health Center & Bole & 31 \\
\hline & $\begin{array}{l}\text { Adisu Gebeya Health } \\
\text { Center }\end{array}$ & Gulele & 22 \\
\hline & Kazanchis Health Center & Kirkos & 19 \\
\hline & Alem Bank Health Center & Kolfe & 38 \\
\hline & $\begin{array}{l}\text { Teklehaymanot Health } \\
\text { Center }\end{array}$ & Lideta & 22 \\
\hline & Woreda 02 Health Center & Nifasilk lafto & 53 \\
\hline & Woreda 13 Health Center & Yeka & 35 \\
\hline
\end{tabular}

$79 \%$ will provide $80 \%$ power to detect a difference between arms (90). The study will recruit and randomly allocate participants meeting the inclusion criteria to intervention and standard DOT arms. Because of the scope of the trial, the study staff and patients will not be blinded to group allocation.

In addition to patients with $\mathrm{TB}$, the study will collect qualitative data from TB healthcare providers to assess further the usability and acceptability of the intervention. For this, the study will include healthcare providers who are currently providing $\mathrm{TB}$ treatment under DOT. The inclusion criteria are: a healthcare provider with academic qualification as a medical doctor, health officer (BSc), or nurse (BSc or Diploma); holds at least 3 months of experience providing DOT services in the facility; and able and willing to provide informed consent. With purposive sampling, the study will enroll 10 healthcare providers, selecting one provider from each of the 10 study sites.

\section{Interventions}

Participants in the intervention arm will receive a 15 day TB medication supply (HRZE fixed-dose combination therapy of 15 doses) in an electronic pillbox device (evriMed500 digital medication monitoring and reminder device manufactured by Wisepill Technologies, South Africa) to self-administer. Providers will collect baseline data-including demographic, socioeconomic, behavioral, and social factors-using the study's baseline questionnaire. Based on the baseline data, participants will be clustered into four behavioral determinants (use cigarettes, alcohol, Khat - a psychostimulant plant, and cocaine/marijuana) and three social determinants (homeless, unemployed, and illiterate) as appropriate for the purpose of analysis. Participants in this arm will return every 15 days, where the provider will count any remaining tablets in the pillbox, download the pill-taking data from the Wisepill device, evaluate the functionality of the device and troubleshoot as needed, and perform the urine isoniazid test. The level of adherence in their intensive phase of treatment will be calculated using the medication possession ratio (MPR) [87]. Any participant who misses more than five tablets in any 15-day refills will be reassigned to daily DOT throughout the remaining days of the intensive phase. Participants in the intervention arm can consult the healthcare provider in cases of medical illness or any adverse events outside of a scheduled visit before the next appointment. The phone number of the healthcare provider following their TB condition will be written at the backside of their appointment cards. The phone call strategy aims to maintain the DOT advantage for the intervention arm.

Participants in the control arm will get their treatment as per the standard practice of DOT, where participants will visit the healthcare facility each business day throughout the 2-month intensive phase to swallow their daily dose of HRZE with direct observation by the healthcare provider. Additionally, they will be given pills for the weekend to take them at home. The provider will collect baseline data, conduct a urine isoniazid test every 15 days, and cluster participants into behavioral and social determinants as applicable for participants in the intervention arm. For this arm, the management of participants who interrupt treatment will follow the national TB treatment guidelines.

Both arms will be followed up throughout the intensive phase which lasts for 2 months. The continuation phase (4 months) will follow the standard DOT practice for both arms. Both arms will have a TB care and treatment service free of charge, and the pillbox will be given to each participant in the intervention arm free of charge. For both arms, participants will receive treatment according to Ethiopian national TB treatment guidelines.

\section{Outcomes \\ Primary outcomes}

The primary outcomes are adherence to medication and sputum conversion.

Level of adherence For this study, the level of adherence will be defined as the proportion of patients who missed doses in intervention (pill count) versus DOT (direct observation) arms, confirmed further by urine isoniazid test (IsoScreen test, GFC Diagnostics Ltd., Bicester, England) [88] and a participant's selfreport of adherence on Morisky Medication Adherence Scale (MMAS-8, an eight-item structured, valid tool to 
measure self-reported adherence to medication) [89]. The time frame will be 2 months, with an assessment of missed doses every day, an IsoScreen urine test every 15 days, and a MMAS- 8 assessment at the end of the 2 months. The WHO defines adherence to medication as the extent to which patients take their medications as prescribed with respect to dosage and intervals throughout the treatment period [90].

Sputum conversion Sputum conversion will be defined as the proportion of patients with smear conversion following the intensive phase in the intervention versus DOT arms, confirmed further by pre-post intensive phase TB liquid culture (BD BACTEC MGIT 960 TB system, BD Diagnostics, MD, US) [91]. Pre-post treatment drug susceptibility testing will determine whether resistance to anti-TB drugs could have impacted culture conversion. The time frame is after the 2-month intensive phase. The updated (2016) Ethiopian National TB guidelines defines sputum conversion as smear conversion to negative after the end of the intensive phase [92].

\section{Secondary outcomes}

Adverse treatment outcomes Adverse treatment outcomes will be defined as the proportion of participants in each arm having one of the following events: treatment not completed; death; or loss to follow-up.

Cost-effectiveness: value of health effects QALYs will be calculated for each participant by arm using the EuroQol's EQ-5D-5 L quality-of-life questionnaire [93], with discount rates of $3 \%$ and a score out of 100 , with 100 defining optimal health. The time horizon will include the entire intensive phase. The questionnaire comprises five dimensions (mobility, self-care, usual activities, pain/discomfort, and anxiety/depression), with each dimension having five close-ended response levels. Average QALYs will be compared by study arm.

Cost-effectiveness: individual and societal costs of treatment Individual and societal costs of treatment will be calculated for each participant using the Tool to Estimate Patients' costs [94]. The Tool is a standard questionnaire developed jointly by KNCV Tuberculosis Foundation, WHO, and the Japan Anti-Tuberculosis Association. It comprises treatment costs (costs related to DOT and picking up TB drugs), guardian costs, and coping costs. The time horizon will include the entire intensive phase. Average costs will be compared by arm.

Acceptability This is the proportion of participants in intervention versus DOT arms who perceived satisfaction with their TB treatment when measured on the Treatment Satisfaction Questionnaire for Medication scale (TSQM v 1.4) administered at the end of the intensive phase. The TSQM is a 14-item valid tool to assess participants' satisfaction with medication across four domains: effectiveness; side effects; convenience; and global satisfaction [95]. The investigators will collect qualitative data from providers to supplement the acceptability study.

Usability Usability will be calculated as the proportion of participants in the intervention arm who perceived the evriMED500 device as usable under adopted System Usability Scale (SUS, a 18-item valid tool to assess user performance of the device in six dimensions: ease of use; challenges; benefits; perceptions of being motivated; popularity; and recommendations) [96]. This assessment will occur at the end of the intensive phase. The investigators will collect qualitative data from providers to supplement the usability study.

\section{Data collection and management}

The study investigators will identify healthcare providers in the TB clinics and provide training on the study procedures, how to operate the evriMED500 device, and how to perform a urine isoniazid test. The investigators will perform a 1:1 randomization of participants before the start of the study using computer-generated random numbers. Providers will enroll participants in the two arms sequentially as they arrive in the clinic and the investigators will routinely monitor the process. Providers will recruit participants and obtain written informed consent in the local language, which is Amharic. For both arms, the providers will collect baseline data, including demographic, socioeconomic, behavioral, and social determinants social using the study's baseline questionnaire. For participants in the intervention arm, the providers will orient participants in the intervention arm as they enroll in how to use the evriMED500 device. The orientation time will depend on the efficiency of the participants to fully acquire and demonstrate the necessary skills. Providers will then dispense a 15-days TB medication supply (HRZE fixed-dose combination therapy of 15 doses) to participants within the evriMed500 device for self-administration. Providers will collect sputum specimens from participants, write their cell phone numbers at the backside of participants' appointment cards to communicate in cases of medical illness or any adverse events before the next appointment, and inform them to return every 15 days. When participants returned, the providers will count any remaining tablets in the pillbox, download the pill-taking data from the evriMED device, check functionally of the device, and conduct a urine isoniazid test. The providers will fill out 
the study's adherence follow-up form to capture information if the drugs are taken every day and, if not, the reasons for non-adherence. The providers will then evaluate the level of adherence based on the preset criteria, and refill a 15-day TB medication supply in the same pillbox as appropriate. The providers will collect sputum specimens from participants at the end of the intensive phase.

For the control arm, providers will handle participants according to the standard DOT procedure, where participants will visit the healthcare facility each business day in the intensive phase to swallow their daily dose of HRZE with direct observation by the provider. The providers will fill out a similar adherence follow-up form for the control arm. Additionally, if a participant in the control arm requests medications for selfadministration, the provider will collect information about the date requested and for how many days requested.

At the end of the intensive phase, trained research experts will complete several data instruments for all participants. The first is a self-report of medication adherence that the experts will administer using the eightitem MMAS-8 questionnaire. The second is a case report form for which data will be extracted from TB registration logs and participants' charts, focusing on overall treatment outcomes and side effects. For the third instrument, the experts will administer the EuroQol's EQ-5D-5 L quality-of-life questionnaire to all participants. The experts will also assess costs using the Tool to Estimate Patients' Costs. Then the experts will administer the TSQM version 1.4 assessment. Finally, the experts will administer the 18-item SUS tool to determine user performance for intervention participants. Following the collection of data from participants, the study investigators will collect qualitative and quantitative data from the providers to learn more about the usability and acceptability of the intervention from providers and the healthcare system perspectives.

For the laboratory research involving biological specimens (urine and sputum), the study will use WHOapproved diagnostic tools. The IsoScreen test is a semiquantitative urine test that provides a reliable and immediate indication of adherence to isoniazid-containing treatment regimens for patients with TB. It uses the reagents of the Arkansas Method-barbituric acid $(20 \mathrm{mg})$, potassium cyanide $(10 \mathrm{mg})$, and chloramine- $\mathrm{T}(10 \mathrm{mg})-$ in an enclosed plastic testing device for safe and rapid testing in clinics and patients' homes. The providers will perform this test within the study facilities for both arms every 15 days, thus four times per participant. Sputum specimens collected before and after the intensive phase will have MGIT TB liquid culture and DST tested at Armauer Hansen Research Institute (AHRI). Initiation, termination, or completion of treatment will rely only on the standard procedures available at the study sites, which could be acid-fast bacilli or Xpert MTB/RIF assay. Thus, the outcomes of the study's laboratory results will not dictate the standard diagnostic or treatment procedures.

The study will use a password-protected offline Research electronic data capture (REDCap) database (Vanderbilt University, Nashville, TN, USA) [97] to enter data and store entered data in an encrypted drive. The Principal Investigator will keep the source data in a locked cabinet at the study's central office. The assigned study staff will check a random sample of $10 \%$ of all data entry forms for entry errors.

\section{Statistical analysis}

The study team will develop a formal statistical analysis plan according to the intention-to-treat principle; the team will develop this plan during the course of the study but prior to a review of any major analyses. The analysis will principally include t-tests, Fisher's exact two-tailed tests, and mixed-effect regression models to determine differences between the intervention and DOT arms. All of the quantitative statistical tests will consider a $p$ value of $<0.05$ to indicate significant associations. For each standardized tool used, their standard scoring algorithm will be normalized for specific demographic characteristics and used for calculations. The qualitative data obtained from individual interviews with healthcare providers will be analyzed using content analysis based on a theoretical framework.

\section{Ethical considerations}

The study obtained formal ethical approval from the Institutional Review Board of the Addis Ababa University College of Health Sciences (ID: 077/19/CDT). The protocol is registered with ClinicalTrials.gov (NCT04216420) and the WHO's International Clinical Trial Registry Platform (ICTRP). Study investigators completed human research participation training and good clinical practice training. All study sites will provide the necessary permissions and written concurrence for data collection. Each participant will provide written informed consent before enrollment. An independent Data and Safety Monitoring Board will oversee the study. Any study staff responsible for the conduct, management, or oversight of the trial will complete good clinical practice training. Any amendment to the protocol will be reviewed and approved by the IRB before the changes are implemented to the study. In addition, all changes to the informed consent form will be IRB-approved; a determination will be made regarding whether a new consent needs to be obtained from participants who provided 
consent using a previously approved informed consent form.

The study does not involve a new investigational product; therefore, no exemption will be sought. The pillbox device has been in use for clinical purposes other than TB. Treatment of TB will not deviate from the national guidelines, neither the type of regimen nor the dose, but only the management of treatment.

Participants will not receive compensation, either in cash or in kind, as this would influence the outcomes of the study. However, if the participants are coming just for the purpose of the study, they will be reimbursed for transport.

\section{Oversite and monitoring}

\section{Interim monitoring and analysis}

The investigators will conduct interim monitoring and submit an analysis report to the independent Data and Safety Monitoring Board (DSMB). Then, the report will be sent together with DSMB's recommendation to the IRBs. The DSMB will periodically review and evaluate the study's collected data to follow up participant safety, the accuracy of study procedures, and the study progress in order to provide recommendations on the continuation, modification, or termination of the study. The DSMB will consist of an expert in the clinical aspects of TB, an expert biostatistician, and an investigator with expertise in current clinical trials conduct and methodology. External monitors may conduct technical audits, before, during, and after completion of the trial. This will include reviewing the research protocol, operations manual, standard operating procedures, and training materials before initiation of the trial. The site investigators will make study documents and pertinent records readily available for inspection by the local IRB and site monitors.

\section{Participant discontinuation}

There will be premature study discontinuation if there is refusal of study participants to participate in all components of the study; a request by the participants to withdraw, request from the healthcare providers if $\mathrm{s} /$ he thinks the study is no longer in the best interest of the participants; or at the discretion of the IRB/Ethics Committee, regulatory bodies, sponsor, or consensus of the investigators.

\section{Unexpected or adverse events}

Given that the study is neither dictating the need for treatment of $\mathrm{TB}$ or specific regimen, serious adverse events will not be directly attributable to the study. The study will not dictate diagnosis or treatment algorithms for $\mathrm{TB}$, and all diagnoses testing assays and treatment regimens will follow the Ethiopian national guidelines; thus, it will not introduce or prescribe new drugs. The major risks of this screening program are related only to pill-taking mechanisms. Adverse reactions to TB medications will not be considered the outcomes of the study. The investigators will capture these events only to the extent they are available in the study health facilities' records and registries. The investigators will conduct chart abstractions to review adverse events that are related to TB treatment, as recorded from the TB clinic registries. For patients who are co-infected with HIV, the chart review will also include the type of HIV regimens, viral load, CD4 counts, and other co-infections diagnosed and treated.

Regarding TB diagnosis, at the initial stage or after the intensive phase, there could be discrepancies between the health facility results (Smear microscopy or Xpert $\mathrm{MTB} / \mathrm{RIF}$ ) and the study result (MGIT liquid culture). In this case, the investigators will communicate the results to the healthcare providers for their review and decision.

\section{Discussion}

This study will be the first in Ethiopia, and of the three in sub-Saharan Africa, to evaluate the effectiveness of pillbox-enabled SAT as a multicenter randomized controlled trial. The study will provide evidence of whether pillbox-enabled SAT is superior to the standard DOT for medication adherence and treatment outcomes. The study will assess whether digital adherence intervention is cost-effective, usable, and acceptable. The study targets patients with TB living in Ethiopia, a high TB burden LMIC in sub-Saharan Africa where alternative TB treatment strategies to in-person DOT do not exist.

If effective, this approach could substantially improve adherence to treatment, increase sputum conversion, reduce patient-side costs due to daily DOT, and improve quality of life. It could also have a strong public health impact by reducing transmission of the disease to healthcare providers and the community at large as the approach can reduce patients' daily travels to healthcare facilities for in-person DOT. This approach will provide patients with TB the freedom and ownership of their treatment, which ultimately reduces mortality and morbidity thereby contributing significantly to the End TB Strategy. The findings will enable patients, healthcare providers, and policymakers to make informed decisions about the value of the intervention.

The study intends to use several clinical, biomedical, behavioral, and economic measurement tools to learn extensively about the desired outcomes of interest. The assessment of adherence using electronic, self-report, and biological specimens will provide credible and reliable justification of results. The diagnostic tools we plan to use are WHO-approved, and the questionnaires are 
valid and psychometrically sound for use in a local context. The study will collect original data from both patients and their TB care providers in local facilities, providing substantial evidence on the usability and acceptability of the intervention in real-world settings.

\section{Trial status}

- Protocol date and version: 4 December 2019, version 3, approved by the Institutional Review Board of the College of Health Sciences, Addis Ababa University on 13 November 2019

- Recruitment start: 1 March 2020

- Recruitment end: 1 February 2021

\section{Supplementary information}

Supplementary information accompanies this paper at https://doi.org/10. 1186/s13063-020-04324-Z.

Additional file 1. SPIRIT 2013 Checklist.

Additional file 2. Participant information sheet and consent form (English version).

\section{Abbreviations}

DAT: Digital adherence technologies; DR-TB: Drug-resistant tuberculosis; DST: Drug susceptibility testing; DOT: Directly observed therapy; HRZE : Isoniazid, rifampicin, pyrazinamide, and ethambutol; MERM: Medication Event Reminder Monitor System; MOST: Multiphase Optimization Strategy; QALY: Quality-adjusted life year; SAT: Self-administered therapy; TB: Tuberculosis; VDOT: Video-supported directly observed therapy; WHO: World Health Organization

\section{Acknowledgments}

We thank Dr. Kidist Bobosha of the Armauer Hansen Research Institute, Ethiopia, for her support with study coordination, and Dr. Janet Gross of the Emory University School of Medicine, USA, for her technical guidance on the study design.

\section{Authors' contributions}

Study conception and Principal Investigator: TM. Initial study design and protocol: TM. Major contribution to study design and protocol: VCM, YW, $\mathrm{DPH}$. Resource acquisition: HMB, AF. Draft the manuscript: TM. Reviewed and revised the manuscript: TM, VCM, YW, DPH, HMB, AF. Read and approved the final manuscript: TM, VCM, YW, DPH, HMB, AF.

\section{Funding}

The trial protocol reported in this publication was supported by the Fogarty International Center and National Institute of Allergy and Infectious Diseases of the U.S. National Institutes of Health under Award Number D43TW009127. VCM received funding from the Emory Center for AIDS Research (P30Al050409). The content is solely the responsibility of the authors and does not necessarily represent the official views of the National Institutes of Health.

\section{Availability of data and materials}

Not applicable.

\section{Ethics approval and consent to participate}

The study obtained formal ethical approval from the Institutional Review Board of the Addis Ababa University College of Health Sciences (ID: 077/19/ CDT). The protocol is registered with ClinicalTrials.gov (NCT04216420). Study investigators completed human research participation training and good clinical practice training. Each participant will provide written consent before enrollment. An independent Data and Safety Monitoring Board will oversee the study.
Consent for publication

Not applicable.

\section{Competing interests}

The authors declare that they have no competing interests.

\section{Author details}

${ }^{1}$ Addis Ababa University, College of Health Sciences, Center for Innovative Drug Development and Therapeutic Trials for Africa, P.O. Box 9086, Addis Ababa, Ethiopia. ${ }^{2}$ Emory University School of Medicine and Rollins School of Public Health, Atlanta, GA 30322, USA.

Received: 16 March 2020 Accepted: 10 April 2020

Published online: 05 May 2020

References

1. Jiang WX, Long Q, Lucas H, Dong D, Chen JY, Xiang L, et al. Impact of an innovative financing and payment model on tuberculosis patients' financial burden: is tuberculosis care more affordable for the poor? Infect Dis Poverty. 2019;8(1):21..

2. Ukwaja KN. Social protection interventions could improve tuberculosis treatment outcomes. Lancet Glob Health. 2019;7(2):e167-8.

3. Richterman A, Steer-Massaro J, Jarolimova J, Luong Nguyen LB, Werdenberg J, Ivers LC. Cash interventions to improve clinical outcomes for pulmonary tuberculosis: systematic review and meta-analysis. Bull World Health Organ. 2018:96(7):471-83.

4. Shete PB, Reid M, Goosby E. Message to world leaders: we cannot end tuberculosis without addressing the social and economic burden of the disease. Lancet Glob Health. 2018;6(12):e1272-3.

5. Dara M, Zachariah R. Hunger and tuberculosis: Two sides of the same coin. Int J Tuberc Lung Dis. 2018;22(6):592.

6. Sakamoto H, Lee S, Ishizuka A, Hinoshita E, Hori H, Ishibashi N, et al. Challenges and opportunities for eliminating tuberculosis - leveraging political momentum of the UN high-level meeting on tuberculosis. BMC Public Health. 2019;19(1):76.

7. Ki-Moon B. Building a tuberculosis-free world on a foundation of universal health coverage. Lancet. 2019;393(10178):1268-70.

8. Agins BD, Ikeda DJ, Reid MJA, Goosby E, Pai M, Cattamanchi A. Improving the cascade of global tuberculosis care: moving from the "what" to the "how" of quality improvement. Lancet Infect Dis. 2019;19:e437-43.

9. Reid MJA, Arinaminpathy N, Bloom A, Bloom BR, Boehme C, Chaisson R, et al. Building a tuberculosis-free world: The Lancet Commission on tuberculosis. Lancet. 2019;393(10178):1331-84

10. Cohen KA, Manson AL, Abeel T, Desjardins CA, Chapman SB, Hoffner S, et al. Extensive global movement of multidrug-resistantM. tuberculosis strains revealed by whole-genome analysis. Thorax. 2019;74(9):882-9.

11. Chihota VN, Niehaus A, Streicher EM, Wang X, Sampson SL, Mason P, et al. Geospatial distribution of Mycobacterium tuberculosis genotypes in Africa. PLoS One. 2018;13(8):e0200632.

12. Knight GM, McQuaid CF, Dodd PJ, Houben RMGJ. Global burden of latent multidrug-resistant tuberculosis: trends and estimates based on mathematical modelling. Lancet Infect Dis. 2019;19(8):903-12.

13. Walker TM, Merker M, Knoblauch AM, Helbling P, Schoch OD, van der Werf MJ, et al. A cluster of multidrug-resistant Mycobacterium tuberculosis among patients arriving in Europe from the Horn of Africa: a molecular epidemiological study. Lancet Infect Dis. 2018;18(4):431-40.

14. Wild V, Jaff D, Shah NS, Frick M. Tuberculosis, human rights and ethics considerations along the route of a highly vulnerable migrant from subSaharan Africa to Europe. Int J Tuberc Lung Dis. 2017;21(10):1075-85.

15. Armstrong LR, Winston CA, Stewart B, Tsang CA, Langer AJ, Navin TR. Changes in tuberculosis epidemiology, United States, 1993-2017. Int J Tuberc Lung Dis. 2019;23(7):797-804.

16. Ismail N, Ismail F, Omar SV, Blows L, Gardee Y, Koornhof H, et al. Drug resistant tuberculosis in Africa: Current status, gaps and opportunities. Afr J Lab Med. 2018;7(2):781.

17. World Health Organization (WHO). Global tuberculosis report 2019. Geneva: WHO; 2019

18. Eshetie S, Gizachew M, Alebel A, van Soolingen D. Tuberculosis treatment outcomes in Ethiopia from 2003 to 2016, and impact of HIV co-infection and prior drug exposure: A systematic review and meta-analysis. PLoS One. 2018;13(3):e0194675. 
19. Matteelli A, Rendon A, Tiberi S, Al-Abri S, Voniatis C, Carvalho ACC, et al. Tuberculosis elimination: where are we now? Eur Respir Rev. 2018;27(148): 180035.

20. Collins D, Hafidz F, Mustikawati D. The economic burden of tuberculosis in Indonesia. Int J Tuberc Lung Dis. 2017;21:1041-8.

21. Tanimura T, Jaramillo E, Weil D, Raviglione M, Lonnroth K. Financial burden for tuberculosis patients in low- and middle income countries: a systematic review. Eur Respir J. 2014;43:1763-75.

22. van den Hof S, Collins D, Hafidz F, Beyene D, Tursynbayeva A, Tiemersma E. The socioeconomic impact of multidrug resistant tuberculosis on patients: results from Ethiopia, Indonesia and Kazakhstan. BMC Infect Dis. 2016;16:470.

23. Harries AD, Schwoebel V, Monedero-Recuero I, Aung TK, Chadha S, Chiang $C Y$, et al. Challenges and opportunities to prevent tuberculosis in people living with HIV in low-income countries. Int J Tuberc Lung Dis. 2019;23(2): 241-51.

24. Diallo A, Dahourou DL, Dah TTE, Tassembedo S, Sawadogo R, Meda N. Factors associated with tuberculosis treatment failure in the Central East Health region of Burkina Faso. Pan Afr Med J. 2018;30:293.

25. Ruru Y, Matasik M, Oktavian A, Senyorita R, Mirino Y, Tarigan LH, et al. Factors associated with non-adherence during tuberculosis treatment among patients treated with DOTS strategy in Jayapura, Papua Province, Indonesia. Glob Health Action. 2018;11(1):1510592.

26. Sharma SK, Jha S. Directly observed treatment is not the only solution for poor adherence and low cure of tuberculosis. Evid Based Med. 2015;20(5):180.

27. Alipanah N, Jarlsberg L, Miller C, Linh NN, Falzon D, Jaramillo E, et al. Adherence interventions and outcomes of tuberculosis treatment: A systematic review and meta-analysis of trials and observational studies. PLoS Med. 2018;15(7):e1002595.

28. DiStefano MJ, Schmidt H. mHealth for Tuberculosis Treatment Adherence: A Framework to Guide Ethical Planning, Implementation, and Evaluation. Glob Health Sci Pract. 2016;4(2):211-21.

29. Elangovan R, Arulchelvan S. A Study on the Role of Mobile Phone Communication in Tuberculosis DOTS Treatment. Indian J Community Med. 2013;38(4):229-33.

30. Lei $X$, Liu Q, Wang H, Tang X, Li L, Wang Y. Is the short messaging service feasible to improve adherence to tuberculosis care? A cross-sectional study. Trans R Soc Trop Med Hyg. 2013;107(10):666-8.

31. Nsengiyumva NP, Mappin-Kasirer B, Oxlade O, Bastos M, Trajman A, Falzon $D$, et al. Evaluating the potential costs and impact of digital health technologies for tuberculosis treatment support. Eur Respir J. 2018;52(5): 1801363.

32. Subbaraman R, de Mondesert L, Musiimenta A, Pai M, Mayer KH, Thomas BE, et al. Digital adherence technologies for the management of tuberculosis therapy: mapping the landscape and research priorities. BMJ Glob Health. 2018;3(5):e001018.

33. Ngwatu BK, Nsengiyumva NP, Oxlade O, Mappin-Kasirer B, Nguyen NL,

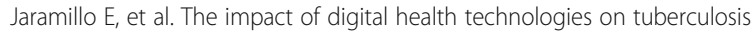
treatment: a systematic review. Eur Respir J. 2018;51(1):1701596.

34. Denkinger CM, Grenier J, Stratis AK, Akkihal A, Pant-Pai N, Pai M. Mobile health to improve tuberculosis care and control: a call worth making. Int J Tuberc Lung Dis. 2013;17(6):719-27.

35. Nglazi MD, Bekker LG, Wood R, Hussey GD, Wiysonge CS. Mobile phone text messaging for promoting adherence to anti-tuberculosis treatment: a systematic review. BMC Infect Dis. 2013;13:566.

36. World Health Organization (WHO). Handbook for the use of digital technologies to support tuberculosis medication adherence. Geneva: World Health Organization; 2017.

37. Bediang G, Stoll B, Elia N, Abena JL, Geissbuhler A. SMS reminders to improve adherence and cure of tuberculosis patients in Cameroon (TB-SMS Cameroon): a randomized controlled trial. BMC Public Health. 2018;18(1):583.

38. Fang XH, Guan SY, Tang L, Tao FB, Zou Z, Wang JX, et al. Effect of Short Message Service on Management of Pulmonary Tuberculosis Patients in Anhui Province, China: A Prospective, Randomized, Controlled Study. Med Sci Monit. 2017:23:2465-9.

39. Mohammed S, Glennerster R, Khan AJ. Impact of a daily SMS medication reminder system on tuberculosis treatment outcomes: a randomized controlled trial. PLoS One. 2016;11(11):e0162944.

40. Liu X, Lewis JJ, Zhang H, Lu W, Zhang S, Zheng G, et al. Effectiveness of electronic reminders to improve medication adherence in tuberculosis patients: a cluster-randomised trial. PLoS Med. 2015;12(9):e1001876.
41. Iribarren S, Beck S, Pearce PF, Chirico C, Etchevarria M, Cardinale D, et al TextTB: A Mixed Method Pilot Study Evaluating Acceptance, Feasibility, and Exploring Initial Efficacy of a Text Messaging Intervention to Support TB Treatment Adherence. Tuberc Res Treat. 2013;2013:349394.

42. Onwubiko U, Wall K, Sales RM, Holland DP. Using Directly Observed Therapy (DOT) for latent tuberculosis treatment - A hit or a miss? A propensity score analysis of treatment completion among 274 homeless adults in Fulton County, GA. PLoS One. 2019;14(6):e0218373.

43. Park S, Sentissi I, Gil SJ, Park WS, Oh B, Son AR, et al. Medication Event Monitoring System for Infectious Tuberculosis Treatment in Morocco: A Retrospective Cohort Study. Int J Environ Res Public Health. 2019;16(3):E412.

44. Liu X, Blaschke T, Thomas B, De Geest S, Jiang S, Gao Y, et al. Usability of a Medication Event Reminder Monitor System (MERM) by Providers and Patients to Improve Adherence in the Management of Tuberculosis. Int J Environ Res Public Health. 2017;14(10):E1115.

45. Broomhead S, Mars M. Retrospective return on investment analysis of an electronic treatment adherence device piloted in the Northern Cape Province. Telemed J E Health. 2012;18(1):24-31.

46. Thakkar D, Piparva KG, Lakkad SG. A pilot project: 99DOTS information communication technology-based approach for tuberculosis treatment in Rajkot district. Lung India. 2019;36(2):108-11.

47. Lam CK, McGinnis Pilote K, Haque A, Burzynski J, Chuck C, Macaraig M. Using Video Technology to Increase Treatment Completion for Patients With Latent Tuberculosis Infection on 3-Month Isoniazid and Rifapentine: An Implementation Study. J Med Internet Res. 2018;20(11):e287.

48. Garfein RS, Liu L, Cuevas-Mota J, Collins K, Muñoz F, Catanzaro DG, et al. Tuberculosis Treatment Monitoring by Video Directly Observed Therapy in 5 Health Districts, California, USA. Emerg Infect Dis. 2018;24(10):1806-15.

49. Nguyen TA, Pham MT, Nguyen TL, Nguyen VN, Pham DC, Nguyen BH, et al. Video Directly Observed Therapy to support adherence with treatment for tuberculosis in Vietnam: A prospective cohort study. Int J Infect Dis. 2017:65:85-9.

50. Chuck C, Robinson E, Macaraig M, Alexander M, Burzynski J. Enhancing management of tuberculosis treatment with video directly observed therapy in New York City. Int J Tuberc Lung Dis. 2016;20(5):588-93.

51. Garfein RS, Collins K, Muñoz F, Moser K, Cerecer-Callu P, Raab F, et al. Feasibility of tuberculosis treatment monitoring by video directly observed therapy: a binational pilot study. Int J Tuberc Lung Dis. 2015;19(9):1057-64.

52. Pérez-Jover V, Sala-González M, Guilabert M, Mira JJ. Mobile Apps for Increasing Treatment Adherence: Systematic Review. J Med Internet Res. 2019;21(6):e12505.

53. Schwartz JK. Pillbox use, satisfaction, and effectiveness among persons with chronic health conditions. Assist Technol. 2017;29(4):181-7.

54. e Souza FR, da Silva Santana C. A descriptive study about the use of pillboxes by older adults. Health. 2013;05(12):103-9.

55. Sanders MJ, van Oss T. Using daily routines to promote medication adherence in older adults. Am J Occup Ther. 2013;67(1):91-9.

56. Hayes TL, Hunt JM, Adami A, Kaye JA. An electronic pillbox for continuous monitoring of medication adherence. Conf Proc IEEE Eng Med Biol Soc. 2006;1:6400-3.

57. Wisepill. Somerset West, South Africa. https://www.wisepill.com/.

58. Wisepill Technologies. evriMED500 medication monitoring and reminder system: introduction, Version: 1.333. Wisepill; South Africa, 2019. https:// static1.squarespace.com/static/56224d8fe4b092a567c227aa/t/5c8a542b4e1 7b635a5f442bf/1552569435055/evriMED500+Product+Handbook+ Ver+1.333+14-03-19.pdf.

59. Mhimbira F, Hella J, Maroa T, Kisandu S, Chiryamkubi M, Said K, et al. HomeBased and Facility-Based Directly Observed Therapy of Tuberculosis Treatment under Programmatic Conditions in Urban Tanzania. PLoS One. 2016;11(8):e0161171

60. Mkopi A, Range N, Lwilla F, Egwaga S, Schulze A, Geubbels E, et al. Adherence to tuberculosis therapy among patients receiving home-based directly observed treatment: evidence from the United Republic of Tanzania. PLoS One. 2012;7(12):e51828.

61. Egwaga S, Mkopi A, Range N, Haag-Arbenz V, Baraka A, Grewal P. Patientcentred tuberculosis treatment delivery under programmatic conditions in Tanzania: a cohort study. BMC Med. 2009;7:80.

62. Oyieng'o D, Park P, Gardner A, Kisang G, Diero L, Sitienei J, et al. Community-based treatment of multidrug-resistant tuberculosis: early experience and results from Western Kenya. Public Health Action. 2012;2(2):38-42. 
63. Nackers F, Huerga H, Espié E, Aloo AO, Bastard M, Etard JF, et al. Adherence to self-administered tuberculosis treatment in a high HIV-prevalence setting: a cross-sectional survey in Homa Bay, Kenya. PLoS One. 2012;7(3):e32140.

64. Cremers AL, Gerrets R, Kapata N, Kabika A, Birnie E, Klipstein-Grobusch K, et al. Tuberculosis patients' pre-hospital delay and non-compliance with a longstanding DOT programme: a mixed methods study in urban Zambia. BMC Public Health. 2016;16(1):1130.

65. Serapelwane MG, Davhana-Maselesele M, Masilo GM. Experiences of patients having tuberculosis (TB) regarding the use of Directly Observed Treatment Short-Course (DOTS) in the North West Province, South Africa. Curationis. 2016;39(1):e1-9.

66. Kaplan R, Caldwell J, Hermans S, Adriaanse S, Mtwisha L, Bekker LG, et al. An integrated community TB-HIV adherence model provides an alternative to DOT for tuberculosis patients in Cape Town. Int J Tuberc Lung Dis. 2016; 20(9):1185-91.

67. Gebreweld FH, Kifle MM, Gebremicheal FE, Simel LL, Gezae MM, Ghebreyesus SS, et al. Factors influencing adherence to tuberculosis treatment in Asmara, Eritrea: a qualitative study. J Health Popul Nutr. 2018;37(1):1.

68. Collins D, Beyene D, Tedla Y, Mesfin H, Diro E. Can patients afford the cost of treatment for multidrug-resistant tuberculosis in Ethiopia? Int J Tuberc Lung Dis. 2018;22(8):905-11.

69. Asres A, Jerene D, Deressa W. Pre- and post-diagnosis costs of tuberculosis to patients on Directly Observed Treatment Short course in districts of southwestern Ethiopia: a longitudinal study. J Health Popul Nutr. 2018;37(1):15

70. Woimo T, Yimer WK, Bati T, Gesesew HA. The prevalence and factors associated for anti-tuberculosis treatment non-adherence among pulmonary tuberculosis patients in public health care facilities in South Ethiopia: a cross-sectional study. BMC Public Health. 2017;17(1):269.

71. Getahun B, Wubie M, Dejenu G, Manyazewal T. Tuberculosis care strategies and their economic consequences for patients: the missing link to end tuberculosis. Infect Dis Poverty. 2016;5(1):93.

72. Mesfin EA, Beyene D, Tesfaye A, Admasu A, Addise D, Amare M, et al. Drugresistance patterns of Mycobacterium tuberculosis strains and associated risk factors among multi drug-resistant tuberculosis suspected patients from Ethiopia. PLoS One. 2018;13(6):e0197737.

73. Mussie KM, Yimer SA, Manyazewal T, et al. Exploring local realities: Perceptions and experiences of healthcare workers on the management and control of drug-resistant tuberculosis in Addis Ababa. Ethiopia. PLoS One. 2019;14(11):e0224277.

74. Fiseha D, Demissie M. Assessment of Directly Observed Therapy (DOT) following tuberculosis regimen change in Addis Ababa, Ethiopia: a qualitative study. BMC Infect Dis. 2015;15:405.

75. Genet C, Melese A, Worede A. Effectiveness of directly observed treatment short course (DOTS) on treatment of tuberculosis patients in public health facilities of Debre Tabor Town, Ethiopia: retrospective study. BMC Res Notes. 2019;12(1):396.

76. Getahun B, Nkosi ZZ. Is directly observed tuberculosis treatment strategy patient-centered? A mixed method study in Addis Ababa, Ethiopia. PLoS One. 2017;12(8):e0181205.

77. Sagbakken M, Frich JC, Bjune GA, Porter JD. Ethical aspects of directly observed treatment for tuberculosis: a cross-cultural comparison. BMC Med Ethics. 2013;14:25.

78. Getahun B, Nkosi ZZ. Satisfaction of patients with directly observed treatment strategy in Addis Ababa, Ethiopia: A mixed-methods study. PLoS One. 2017:12(2):e0171209.

79. Adenager GS, Alemseged F, Asefa H, Gebremedhin AT. Factors Associated with Treatment Delay among Pulmonary Tuberculosis Patients in Public and Private Health Facilities in Addis Ababa, Ethiopia. Tuberc Res Treat. 2017; 2017:5120841.

80. Tadesse S. Stigma against Tuberculosis Patients in Addis Ababa, Ethiopia. PLoS One. 2016;11(4):e0152900

81. Tola HH, Holakouie-Naieni K, Tesfaye E, Mansournia MA, Yaseri M Prevalence of tuberculosis treatment non-adherence in Ethiopia: a systematic review and meta-analysis. Int J Tuberc Lung Dis. 2019;23(6):741-9.

82. Tesfay K, Tesfay S, Nigus E, Gebreyesus A, Gebreegziabiher D, Adane K. More than half of presumptive multidrug-resistant cases referred to a tuberculosis referral laboratory in the Tigray region of Ethiopia are multidrug resistant. Int J Mycobacteriol. 2016;5(3):324-7.
83. Tafess K, Beyen TK, Abera A, Tasew G, Mekit S, Sisay S, et al. Treatment Outcomes of Tuberculosis at Asella Teaching Hospital, Ethiopia: Ten Years' Retrospective Aggregated Data. Front Med (Lausanne). 2018;5:38.

84. World Health Organization. WHO End TB Strategy. Geneva: WHO; 2015.

85. Chan AW, Tetzlaff JM, Gøtzsche PC, et al. SPIRIT 2013 explanation and elaboration: guidance for protocols of clinical trials. BMJ. 2013;346:e7586.

86. Ethiopian Federal Ministry of Health. Health and health related indicators. Addis Ababa: FMoH; 2016.

87. Kozma CM, Dickson M, Phillips AL, et al. Medication possession ratio: implications of using fixed and variable observation periods in assessing adherence with disease-modifying drugs in patients with multiple sclerosis. Patient Prefer Adherence. 2013;7:509-16.

88. GFC Diagnostics Itd. IsoScreen test. Oxfordshire, UK. http://www. gfcdiagnostics.com/isoscreen.html.

89. Morisky DE, DiMatteo MR. Improving the measurement of self-reported medication nonadherence: final response. J Clin Epidemio. 2011;64:258-63.

90. World Health Organization. Adherence to long-term therapies: evidence for action. Geneva: WHO; 2003.

91. Becton, Dickinson and Company. BD BACTEC ${ }^{\mathrm{TM}}$ MGIT'M automated mycobacterial detection system. Maryland, US. https://www.bd.com/en-us/ offerings/capabilities/microbiology-solutions/mycobacteria-testing/bdbactec-mgit-automated-mycobacterial-detection-system.

92. Ethiopian Federal Ministry of Health (FMoH). National comprehensive tuberculosis, leprosy and TB/HIV training manual for health care workers. Addis Ababa: FMoH; 2016.

93. EQ 5 D. EQ-5D-5 L User Guide: basic information on how to use the EQ-5D$5 \mathrm{~L}$ instrument, Version 2.1. Rotterdam: EQ. 5D; 2015.

94. TB CAP. Tool to Estimate Patients Costs. Hague: TB CAP; 2008. http://www. stoptb.org/wg/dots_expansion/tbandpoverty/spotlight.asp.

95. Atkinson MJ, Sinha A, Hass SL, Colman SS, Kumar RN, Brod M, et al. Validation of a general measure of treatment satisfaction, the Treatment Satisfaction Questionnaire for Medication (TSQM), using a national panel study of chronic disease. Health Qual Life Outcomes. 2004;2:12.

96. Brooke J. The System Usability Scale. USA: Hewlett-Packard; 1986.

97. Wright A. REDCap: A tool for the electronic capture of research data. J Electron Resour Med Libr. 2016;13:197-201.

\section{Publisher's Note}

Springer Nature remains neutral with regard to jurisdictional claims in published maps and institutional affiliations.
Ready to submit your research? Choose BMC and benefit from:

- fast, convenient online submission

- thorough peer review by experienced researchers in your field

- rapid publication on acceptance

- support for research data, including large and complex data types

- gold Open Access which fosters wider collaboration and increased citations

- maximum visibility for your research: over $100 \mathrm{M}$ website views per year

At BMC, research is always in progress.

Learn more biomedcentral.com/submissions 